\title{
Discrete dipole approximation simulation of optical vortex excited plasmonic properties of a partially capped core-shell nanostructure
}

\author{
Daisuke Tanaka*a, Shungo Harajiri ${ }^{\mathrm{a}}$, David Andrews ${ }^{\mathrm{b}}$, Kayn A. Forbes ${ }^{\mathrm{c}}$ \\ ${ }^{a}$ National Institute of Technology (NIT), Oita College, 1666 Maki, Oita, Japan; \\ ${ }^{b}$ Chemistry, University of East Anglia, Norwich Research Park, Norwich, Norfolk, NR4 7TJ, UK; \\ 'Nanostructures and Photomolecular Systems Group School of Chemical Sciences, University of \\ East Anglia, Norwich Research Park, Norwich, NR4 7TJ, UK
}

\begin{abstract}
In this study, interactions between optical vortex light and partially capped core-shell (PCCS) nanostructures simulated by a discrete dipole approximation (DDA) method are described. The plasmonic characteristics of the PCCS structure, in which a part of the surface of dielectric nanoparticles is covered with metal cap, can be tuned by the core-shell ratio and the coverage condition. It was found that the topological charge and the polarization degree indicating the state of the optical vortex determine the resonance order, peak wavelength, direction and magnitude of optical torque of the excited pseudo-plasmon resonance of PCCS structures. It was also found that under certain incident conditions, higher-order resonance modes, such as hexapole and octupole modes are excited. Likewise, distorted resonance modes due to the asymmetry of the structure are excited. These plasmonic characteristics cannot be realized by scalar beam excitation, it becomes possible by an asymmetrical PCCS structure with optical vortex excitation.
\end{abstract}

Keywords: Discrete dipole approximation, Optical vortex, Asymmetric nanostructure, Localized plasmon resonance

*d-tanaka@oita-ct.ac.jp; phone +81-97-552-6992; fax +81-97-52-7144

\section{INTRODUCTION}

Recently, singular optical properties of metallic nanostructures derived from localized plasmon resonances (LPRs) have been gaining much attention. LPR is a resonant phenomenon that occurs between the light waves and plasma oscillations of electrons (plasmons), and can be induced by light irradiation without the need for optical elements such as optical prisms. At the resonant condition, optical electric fields near the surface of the nanostructure are greatly enhanced by confinement effect of the incident light wave in a region narrower than the diffraction limit and the optical absorption and scattering have maximum at the plasmon resonant frequency. The resonance condition depends on polarization degree of incident light, size, shape, and material of the structure, and refractive index of ambient medium. Further, unique optical properties due to multiple pole resonance modes, which are excited by complex nanostructures, and complex resonance modes excited by their mode interference (Fano resonance and plasmon hybridization) have also been reported $[1,2]$. These multipole plasmon modes such as quadrupole typically show lower radiative losses and higher quality factors than the dipole plasmon resonance and are hence referred to as dark mode resonances. Most of the papers about LPRs have reported unique optical properties due to the complexity of nanostructures, and there are few papers, which have paid attention to the tuning of optical properties by controlling the excitation light [3]. In 2015, Sakai et al. reported the results of optical simulations by using commercial software COMSOL that showed the pseudo highorder localized plasmon resonance can be excited by an optical vortex beam [4]. The paper described that the order of the resonance mode can be controlled by the condition of the incident optical vortex beam, and that the resonance mode rotates with time. An optical vortex is one of the vector beam different from general scalar beams. In an optical vortex, the phase plane is twisted around its axis when the beam is propagating in the space. We represented the orbital angular momentum, which corresponds to number of twists in one wavelength, by the topological charge $l$ and the spin angular momentum, which corresponds to the direction of polarization (right circular polarization or left circular polarization), by $\sigma$. The number $l$ is always an integer, and can be positive or negative, depending on the direction of the twist. Because of the twisting, the light waves at the axis itself cancel each other out. Therefore, when the incident light enters a flat surface, the intensity distribution of the optical vortex looks like a ring, with a dark hole in the center. A vector beam 
such as the optical vortex has some attractive elements as an orbital angular momentum or an electric field to the propagation direction. Therefore, LPR excitation by vector beams has a potential to pave the new way of the plasmonics.

In this study, we investigated the optical characteristics when an optical vortex beam of RPC or LPC are incident to single partially capped core-shell (PCCS) structure in which a part of dielectric nanoparticles is covered with metal by the discrete dipole approximation (DDA) simulation. The plasmonic characteristics of the PCCS structure can be tuned by the core-shell ratio and the coverage condition. In addition, since a functional material such as luminescence material or non-linear optical material can be selected for the core, it can be said that the structure has high versatility.

\section{DDA SIMULATION AND CALCULATION MODEL}

The DDA method is a numerical simulation method that can calculate the light absorption, light scattering, and near field intensity, of the structure, which is regarded as an aggregate of electric dipoles, by assuming that the dipole at position $r_{i}$ has a moment $p_{i}$, which is expressed the following equation (1), when the external electric field $E_{\text {ext }}$ is induced to the structure.

$$
p_{i}=\alpha E_{l o c}\left(r_{i}\right)
$$

Where, $E_{l o c}$ is the local electric field at $r_{i}$, and $\alpha$ is the polarizability of the dipole. DDSCAT [5, 6] was used to simulate and rewrote the part of the calculation codes to set optical vortex beams as the incident light. DDSCAT describes the state of the polarization of the incident light by Jones calculus. Therefore, we can define the optical vortex beam as incident light by rewriting to the following equation (2).

$$
\left(\begin{array}{l}
E_{x} \\
E_{y} \\
E_{z}
\end{array}\right)=e^{-i \varphi}\left(\begin{array}{l}
0 \\
1 \\
i
\end{array}\right)
$$

Where $\varphi$ is the azimuth angle of the electric field oscillating in the $y z$ plane after passing through an axisymmetric polarizing element, and $i$ is the imaginary unit in the case that the incident light propagates in the $x$ direction. Equation (2) expresses the counterclockwise circularly polarized optical vortex, and it can be changed to clockwise circularly polarized light by changing the sign of $z$ components. In this study, the vortex beam of RCP or LCP is set as the incident light with $l=-1,-2,-3$, and -4 .

We observed the extinction spectra and the electric field distribution of PCCS particles at peak wavelengths under the following conditions. The diameter of the core nanosphere and the thickness of the metal shell were fixed at $100 \mathrm{~nm}$ and $10 \mathrm{~nm}$, respectively. The metal thin film covered half of the nanospheres, and the film thickness was set to uniform everywhere. The surrounding medium was a vacuum, and core material and shell metal were chosen the silica and gold. The refractive index of silica was fixed to 1.45 without attenuation coefficient, ignoring the dispersion. The dielectric function of gold was referred from the study by Johnson and Christy [7], neglecting the size dependence of scattering electron. The dipole element spacing of the PCCS structure is fixed to $2 \mathrm{~nm}$ in all calculation models.

\section{RESULT AND DISCUSSION}

\subsection{Extinction spectra and electric field distribution}

Figure 1 show the extinction spectra of single PCCS particle when the RCP optical vortex beam with each topological charge from -1 to -4 . The direction of incidence was set to the wave vector $k$ penetrates the metal nanocap, as shown in the inset. The insets in the graphs are the electric field distribution maps at each peak wavelength in the $y z$ plane at the center of the nanoparticles. The extinction spectra has two peaks, one is the sharp peak at around 550-700 nm the other is the broad peak around $1600 \mathrm{~nm}$. Focusing on the peak wavelengths at short range, the peak value of the extinction spectra decrease as the topological charge decreases, and the resonance wavelength shifts to the short wavelength side. On the contrary, the maximum value of the peak on the long wavelength side increases as the topological charge decreases, but the peak wavelength hardly moves. Looking at the electric field distribution map, the order of the resonance mode increases as $l$ decreases. The electric field distribution maps at the wavelength with the maximum peak value are used as inserts, and the order of the resonance modes are the same at other peak wavelengths. It can be seen 
that the resonance mode at any peak wavelength shows the centrosymmetric multipole resonances, and the order depends on $l$. From the above, it was found that the excitation efficiency decreases as the order of the resonance mode increases on the short wavelength side, and the opposite occurs on the long wavelength side.

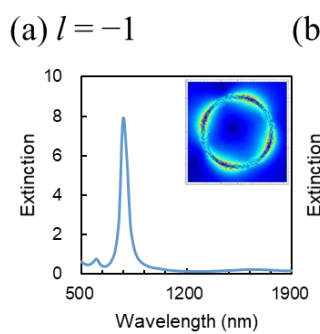

(b) $l=-2$

(c) $l=-3$
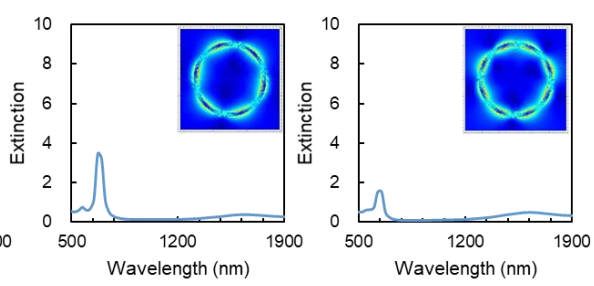

(d) $l=-4$

Figure 1. Simulated extinction spectra and electric field distribution maps of single PCCS particle in the case that RCP $(\sigma=-1)$ optical vortex beams with different $l$ are incident with direction that the wave vector $k$ penetrates the metal nanocap.

Figure 2 shows the extinction spectra and the electric field distribution maps when the LCP optical vortex is incident. Other conditions are the same as in Figure 1. In this case as well, the order of the resonance mode increases monotonically as $l$ decreases. When $l=-1$, a mode that can be expressed as non-polar resonance is excited. The excitation efficiency is maximum when the quadrupole is excited. This simulated results suggest that the non-pole and dipole type resonance have low excitation efficiency, and that the excitation efficiency decreases as the mode order increases in dark mode. If the right circular polarization is expressed by $\sigma=-1$, and the left circular polarization is expressed by $\sigma=+1$, the order of the resonance mode matches $|l+\sigma| \times 2$. These results indicate that the resonance wavelength and excitation efficiency can be controlled by the topological charge $l$ and the direction of polarization.

(a) $l=-1$

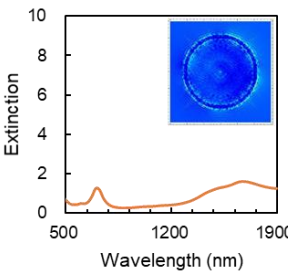

(b) $l=-2$

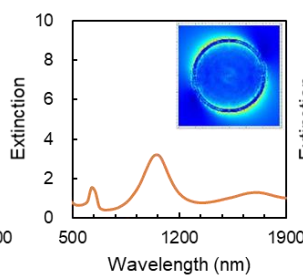

(c) $l=-3$

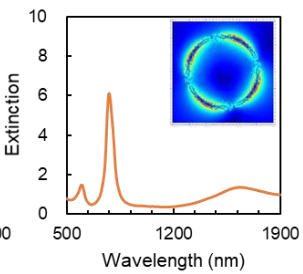

(d) $l=-4$

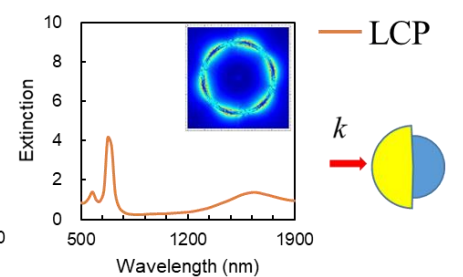

Figure 2. Simulated extinction spectra and electric field distribution maps of single PCCS particle in the case that LCP $(\sigma=1)$ optical vortex beams with different $l$ are incident with direction that the wave vector $k$ penetrates the metal nanocap.

Figure 3 shows the phase dependence of the photoelectric field distribution at the resonant wavelength of the PCCS nanoparticle when the RCP $(\sigma=-1)$ optical vortex with $l=-1,-2,-3$ are incident. Since the phase dependence can be considered by replacing the time dependence, it can be seen that the resonance mode is rotating over time. The intensity at the highest point is almost same in all cases. This distribution profiles cannot be seen in the case of excitation by a scholar beam. The resonance mode excited by a linear polarized light is dipole mode and the position of each poles is fixed in all time (not shown here). This tendency is similar to the case of gold nanodisk [4, 8]. While the phase of the light wave changes from $0 \mathrm{deg}$. to $180 \mathrm{deg}$., the resonance electric fields of $l=-1$ to -3 rotate $90 \mathrm{deg}$., $60 \mathrm{deg}$., and 45 degree, respectively. The resonance field rotates by $180 \mathrm{deg} . /|l+\sigma|$. For example, the angular frequency $\omega$ of the resonance mode is $1.18 \times 10^{15} \mathrm{rad} . / \mathrm{sec}$. in the case that $l=-1$.

Figure 4 shows the simulated extinction spectra when the direction of the wave number of the incident light and the edge of the gold nanocap are parallel. Fig.4 (a)-(d) are in the case of RCP, and (e)-(h) are in the case of LCP. When the incident angle on the PCCS structure changes by 90 degree, the point-symmetrical resonance modes shown in Fig. 1 and Fig. 2 are not excited. Therefore, prediction of the order of resonance modes from $l$ and $\sigma$ is difficult against the distorted resonance modes, tripole-like modes shown in (a) and (b) and the quadrupole-like modes shown in (c) and (d). However, it can be seen that the order of modes tends to increase as $|l+\sigma|$ increases. In the case of RCP incident, the excitation efficiency is low on both the short wavelength side and the long wavelength side. In addition, as $l$ decreases, the maximum peak value on the short wavelength side decreases, and the maximum peak on the long wavelength side increases. The tendency of the peak decrease on the short wavelength side and the peak increase on the long wavelength 
side is the same when LCP is incident as shown in (e)-(f). There are multiple peaks both when RCP is incident and when LCP is incident, and there are cases where five peaks appear as shown in (a) and (g).
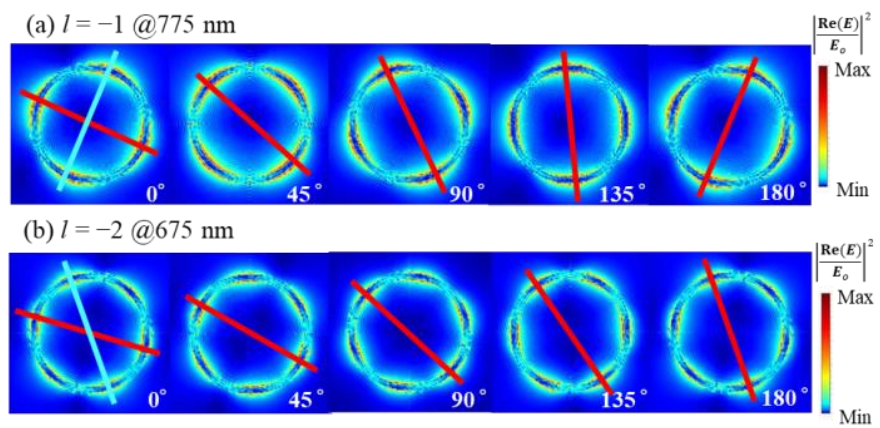

(c) $l=-3 @ 650 \mathrm{~nm}$

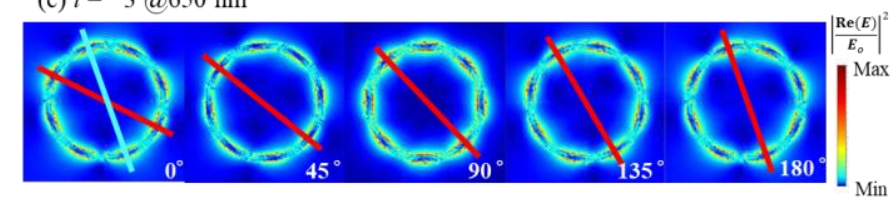

Figure 3. Transition of resonance modes with respect to the phase change of incident light. The $l$ are (a) -1 , (b) -2 , and (c) -3 and $\sigma$ is -1 , respectively. The direction of the wave vector $k$ is perpendicular to the edge of the nanocap of the PCCS nanoparticle.

(a) $l=-1$, RCP

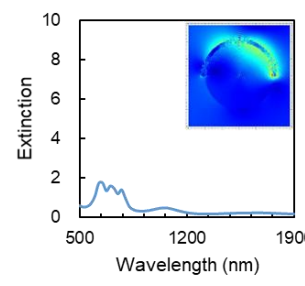

(e) $l=-1$, LCP

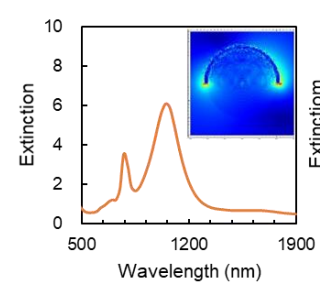

(b) $l=-2$, RCP

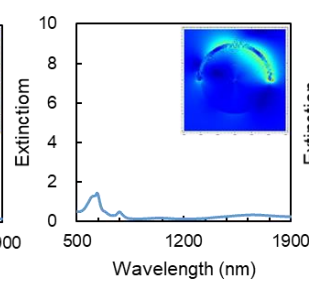

(c) $l=-3$, RCP

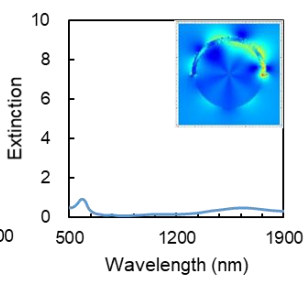

(d) $l=-4$, RCP

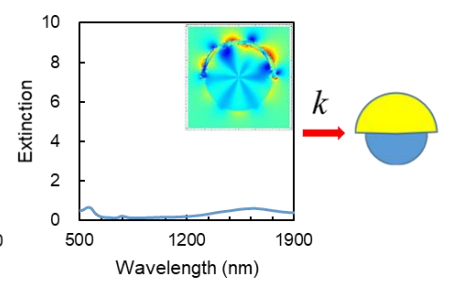

(f) $l=-2, \mathrm{LCP}$

(g) $l=-3$, LCP

(h) $l=-4$, LCP
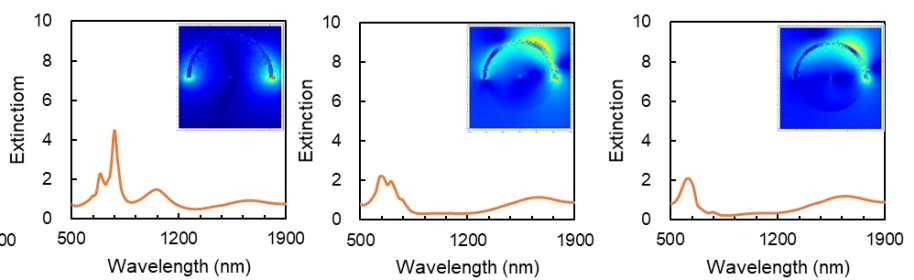

Figure 4. Simulated extinction spectra and electric field distribution maps of single PCCS particle in the case that (a)-(d) RCP $(\sigma=-1)$ and (e)-(h) LCP $(\sigma=1)$ optical vortex beams with different $l$ are incident with direction that the wave vector $k$ and the edge of the nanocap are parallel.

Figure 5 show the phase dependence of the photoelectric field distribution at $781 \mathrm{~nm}$ when the LCP $(\sigma=1)$ optical vortex beam with $l=-2$ is incident to the PCCS nanoparticle in parallel against the edge of the nanocap. In this case, the electric field intensity at the enhancement point changes according to the phase, and shows a tendency similar to the phase dependence when a scalar beam is incident. In other words, this result suggests that this excited mode is different from the resonance derived from the orbital angular momentum and spin angular momentum of the optical vortex. It can be seen that when the structure is asymmetric with respect to the electric field of the incident optical vortex, the pseudo LPR in which the angular momentum of the optical vortex transitions to the nanostructure is not excited. However, the excitation efficiency is not extremely low compared to the case where the structure is symmetric with respect to the incident electric field (see Fig. 4 (e)-(h)). For more detailed analysis, we decided to compare the three-dimensional electric field distribution maps for each incident direction. 

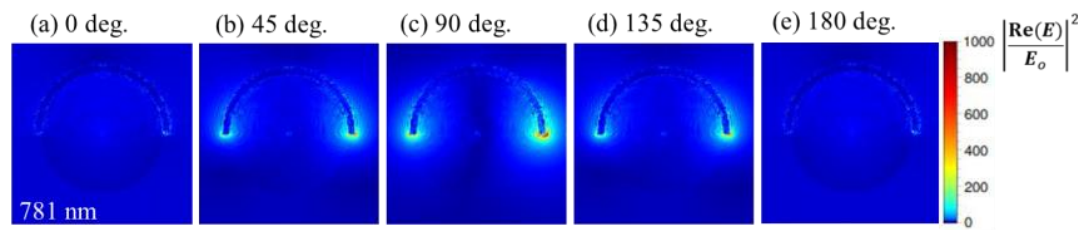

Figure. 5 Transition of resonance mode with respect to the phase change of incident light. The $l$ and $\sigma$ are -2 and 1 , respectively. The wavelength is $781 \mathrm{~nm}$. The direction of the wave vector $k$ is parallel to the edge of the nanocap of the PCCS nanoparticle.

\subsection{Three dimensional maps of electric field distribution}

Figure 6 shows the shows a three-dimensional electric field distribution map observed to confirm the overall picture of the resonance field. Against the edge of the nanocap, the wave vector $k$ is perpendicular in (a) and parallel in (b), respectively. Topological charge $l$ and spin $\sigma$ were set to -2 and 1 in (a), -4 and -1 in (b), respectively. In the incident condition (a), the resonance electric field is distributed in a ring shape at the boundary between the core and the cap, and this result is very similar to the case of the full-shell structure in which the core nanoparticle is fully covered with metal thin film (The result is omitted). In this case, the electric field is localized in a very narrow region, and the enhancement factor is very large, about 1000 times at the maximum. On the other hand, under the incident condition (b), the resonance field is distributed in a band shape on the surface of the metal cap, and it is difficult to determine the number of resonance order from the cross-sectional electric field distribution map. Rather than being localized at a specific position in the nanoparticles, the electric field is widely distributed throughout the nanoparticles, and the enhancement factor is not so large (about 10 times).

(a)

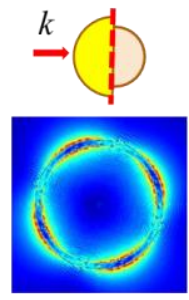

(b)

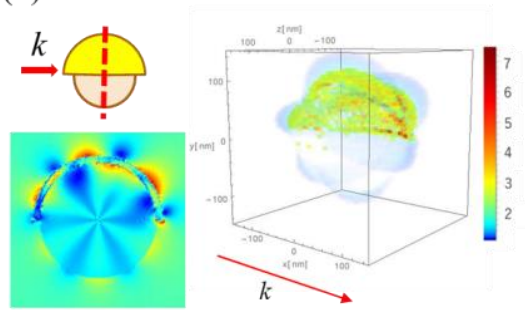

Figure 6. 3D electric field distribution maps of single PCCS particle in the case that the optical vortex beam of (a) LCP with $l=-2$ and (b) RCP with $l=-4$ is incident with different directions, (a) parallel and (b) perpendicular to the edge of the nanocap.

\subsection{Temporal transition of order of LPR modes}

Figure 7 show the resonance field distribution map of a PCCS nanoparticles when the optical vortex of $l=-2$ and $\sigma=-$ 1 is incident parallel to the edge of the nanocap, and ,the phase of the light changes by 45 degree. The resonance mode expected from $|l+\sigma|$ is hexapole, but when the phase is 0 deg., the quadrupole type resonance is excited. After that, it was found that the hexapole type is excited as the phase changes, but returns to the quadrupole type when the phase changes further. Fig. 7 shows the distribution map of the square of the real part of the electric field. When the real part is the hexapole type, the quadrupole type is excited in the imaginary part. If light emission using this mode transition is realized, femtosecond optical modulation will be possible without other optical tools.

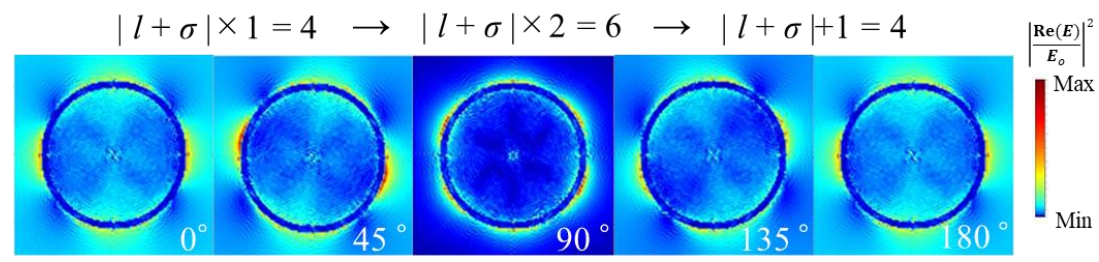

Figure 7. Transition of resonance mode with respect to the phase change of incident light. The $l$ and $\sigma$ are -2 and -1 , respectively. The wavelength is $1610 \mathrm{~nm}$. The direction of the wave vector $k$ is parallel to the edge of the nanocap of the PCCS nanoparticle. 


\subsection{Optical torque excited by the optical vortex beam}

Since the orbital angular momentum $l$ of the incident optical vortex has a great influence on the quasi plasmon resonance mode and the distribution of the resonance electric field, we considered that this orbital angular momentum may add optical torque to the PCCS nanoparticle. Figure 8 shows the radiation torque spectra when a linearly polarized light vortex with $l=-2$ is incident. Since the incident direction is set so that the $k$ vector and the edge of the nanocap are parallel, the distorted resonance mode is excited. A linearly polarized optical vortex can be generated by adding righthanded circularly polarized light and left-handed circularly polarized light, this incident condition corresponds to the simultaneous incidence of both RCP and LCP optical vortices. Here, the positive and negative torques represent whether the torque is clockwise or counterclockwise with respect to each axis. Focusing on the wavelengths of the blue line (690 $\mathrm{nm})$, pink line $(775 \mathrm{~nm})$, and green line $(160 \mathrm{~nm})$ inserted in the graph, it can be seen that the magnitude and the direction of the radiative torque for each axis are different at each wavelength.

This simulation indicates that when a optical vortex of a specific wavelength is incident on a PCCS nanoparticle, the direction and magnitude of the generated torque can be selected by wavelength, and the photodynamic action with threedegree-of-freedom depended on the incident light conditions. For example, when used this PCCS nanoparticle as a nanomotor, it is possible to realize a rotation with three-dimensional-degree-of-freedom. Nanomotors using plasmonic structures have been proposed [9, 10], but motors with three-dimensional-degrees-of-freedom have not been reported, and there is a possibility for new devices.

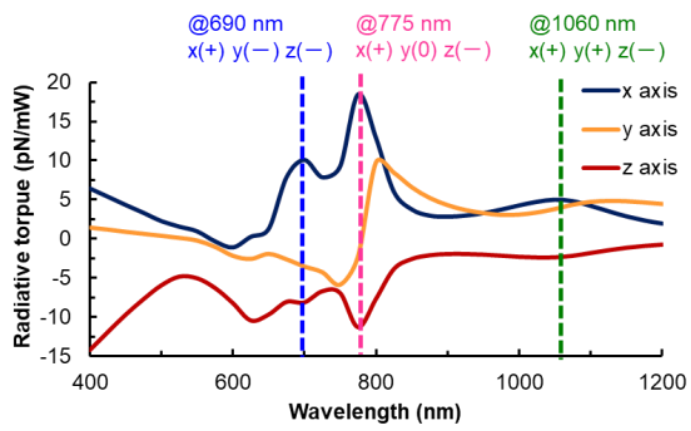

Figure 8. Simulated torque spectra against the each axes. The $l$ was set to -2 , and the vortex beam was set to linear polarization.

\section{CONCLUSION}

We have simulated the optical properties of a PCCS nanoparticles by use of the modified DDSCAT, the LPRs was excited by RCP or LCP optical vortex beams with each topological charge. It was found that in the optical vortex excitation of the PCCS nanoparticle, the pea wavelength and shape of extinction spectra changes depending on the parameters $l$ and $\sigma$, which corresponds to orbital angular momentum and spin angular momentum, and the incident direction of light. Similarly, it was confirmed that the resonance mode when light is incident so as to penetrate the nanocap depends on the $l$ and $\sigma$ of the incident optical vortex. On the other hand, it was also confirmed that it is difficult to expect the order of the resonance mode from the angular momentum because the resonance mode may or may not change depending on the angular momentum when it is incident from the direction perpendicular to the direction through the cap. In addition, we confirmed the difference in the distribution of the resonance field (distributed on the edge of the cap or the surface of the cap) and the difference in the temporal change of the resonance field (rotation or only the intensity changes) depending on the incident direction of light. Furthermore, it was revealed that the resonance mode changes with time under certain conditions. It was also confirmed that plasmon resonance by optical vortex excitation generates torque with three-dimensional degrees of freedom. This result is an interesting finding on higher-order beamexcited plasmon resonance, which is useful for designing new plasmonic devices. 


\section{ACKNOWLEDGEMENT}

This work was partially supported by JSPS KAKENHI Grant-in-Aid for Early-Career Scientists (19K15467) and Nagamori Foundation (Research Grant 2020).

\section{REFERENCES}

[1] M.Hentschel, M. Saliba, R. Vogelgesang, H. Giessen, A. P. Alovosasos and N. Liu: Transition from isolated to collective modes in plasmonic oligomers, Nano Lett., 10(2010), 2721-2726.

[2] N. Verellen, Y. Sonnefraud, H. Sobhani, F. Hao, V. V. Moshchalkov, P. V. Dorpe, P. Nordlander and S.A.Maier: Fano resonances in individual coherent plasmonic nanocavities, Nano Lett., 9(2009), 1663-1667.

[3] M.Hentschel, J. Dorfmuller, H. Giessen, S. Jager, A.M. Kern, K. Braun, D. Zhang and A. Meixner: Plasmonic oligomers in cylindrical vector light beams, Beilstein J. Nanotechnol., 4(2013), 57-65.

[4] K. Sakai, K. Nomura, T. Yamamoto, and K. Sasaki: Excitation of Multipole Plasmons by Optical Vortex Beams, Sci. Rep., 5(2015), 8431

[5] B. T. Draine and P. J. Flatau: Discrete-dipole approximation for scattering calculations, J. Opt. Soc. Am. A, 11(1994), 1491-1499.

[6] B. T. Draine and P. J. Flatau: Discrete-dipole approximation for periodic targets: theory and tests, J. Opt. Soc. Am. A, 25(2008), 2693-2703.

[7] P. B. Johnson and R. W. Christry: Optical constants of the noble metals, Phys. Rew. B, 6(1972), 4370-4379.

[8] S. Harajiri and D. Tanaka: Discrete Dipole Approximation Simulation of LPR Properties of Single Metal Nanodisk Excited by Optical Vortex Beam, Transaction of the Japan Society for Simulation Technology, 12(2020), 21-27.

[9] M. Liu, T. Zentgaf, Y. Liu, G. Bartal, and X. Zhang: Light-Driven nanoscale plasmonic motors, Nat., Nanotechnology, 5(2010), 570-573.

[10] L. Xu, F. Mou, H. Gong, M. Luo, and J. Guan: Light-driven micro/nanomotors: from fundamentals to applications, Chem. Soc. Rev., 46(2017), 6905-6926. 